\section{World's first shark tooth implant for humans}

The Beyond 3000 Dental Clinic in London's Harley Street is offering patients a pair of regenerative dental implants derived from live sharks bred in aquariums in Japan.

Each pair of Scheider Shark Tooth Implants is extracted from a live shark, couriered to London and specially adapted and cut to size in the Beyond 3000 Dental Clinic to fit an individual human patient. One of the implants is inserted into the alveolus in line with the patient's other teeth with the second implant immediately distal to the first. This is necessary because if the more mesial tooth

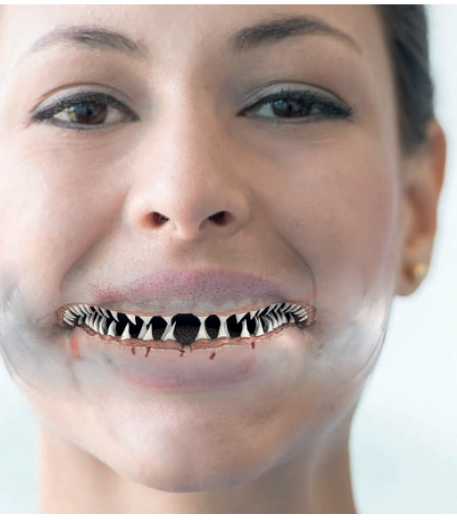
is damaged or knocked out, the distal tooth will move forward into the space mimicking the 'conveyor belt' mechanism that naturally occurs in sharks. As the front implant is unlikely to be used for biting and tearing flesh by humans to the extent seen in sharks (therefore there is no reason that it will be shed in the patient's lifetime, if oral hygiene is maintained), it is expected that the second implant will be a lifetime reserve rather than an active tooth. Patients will be given close instruction in oral hygiene and maintenance of their Scheider Shark Tooth Implants.

Scientists in Japan are currently conducting experiments on teeth from alligators, an animal species that is also able to grow infinite teeth, to see whether they could also be transplanted into humans. Humans possess a similar gene to both sharks and alligators but this is 'switched off' once human adult teeth have erupted and the epithelial cells needed are no longer generated.

To find out more about current offerings at the Beyond 3000 Dental Clinic - also renowned for its TongueShaping treatment and Palatine Uvula Gem Studs, visit www.dreamdental3000.com.

\title{
Harness the power of tooth grinding
}

Do you have patients who grind their teeth? Do you encourage them to wear a mouth guard? Do you suggest they use an electric toothbrush? Do they appear to have high levels of self-reported stress?

If this sounds like a conversation you have with patients, then the Bruxbrush is for you.

The world-leading technology harnesses the energy a patient's mouth produces in a specially designed mouthguard that can then be used to charge up their electric toothbrush. It comes with a carry case and USB connection, designed to make the most of long and tedious car journeys.

Several studies have shown that one eight-hour sleep session with $73.2 \%$ of that involving teeth-grinding can charge an electric toothbrush for up to one hour. Tests have shown that the Bruxbrush can be used in the patient's place of work. If the patient has an irritable colleague, a long meeting or a stressful day at work, tests highlighted a significantly increased energy production delivered to the mouthguard.

\section{If the cap fits}

Providing your patients with the optimum shade through whitening products alone can be an uphill struggle, particularly when regulations limit strengths in whitening gels and chairside treatments.

That's why SoWhiteIt'sBlue is introducing Whiter Cap, a product that flies in the face of hydrogen peroxide safety limits by utilising the natural power of UV to augment your patients' beautifully whitened teeth.

The simple mechanism uses a UV light in patented 'hat form' to deliver a constant beam of safe ultraviolet rays to highlight smiles all day! The invisible electromagnetic radiation beam is attached to the peak area of the patient's cap and as soon as the patient smiles it acts as

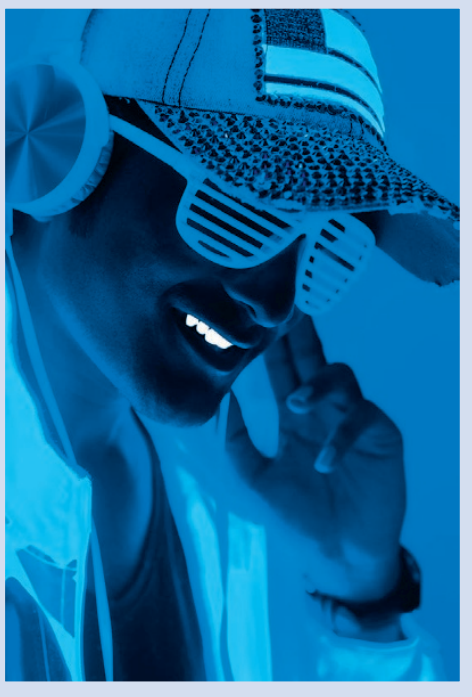
a catalyst to breakdown the whitening peroxide to speed up the whitening process producing that SoWhiteIt'sBlue smile we all know and love.

The baseball cap itself is made from breathable, waterproof material for easy indoor and outdoor use and is available in a wide range of appealing colours to match any outfit. The safe UV light can simply be clipped on each morning as soon as the patient wakes up. A whopping $0.8 \%$ of consumers surveyed were happy to wear the Whiter Cap hat at all times.

So when A1, or even B1 isn't enough for your patients why not suggest Whiter Cap to 'Make Your Teeth White Again!'

Look out for Whiter Hipster Beanie, Whiter Stetson and Whiter Bowler coming soon to the SoWhiteIt'sBlue UV Hat range.

Email queries@SoWhiteIt’sBlue.com or visit www.Whiter-Cap(it's-safe-honestly).com, for more information.

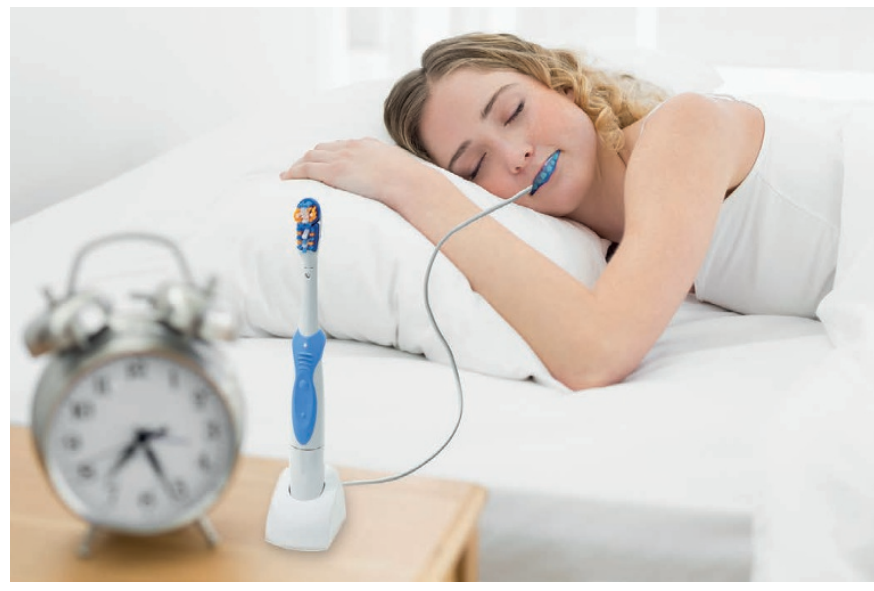

Get your grinding patients going today!

Visit www.bruxbrushgrinder.co.uk. 